\title{
Microbial Aetiology, Antibiotic Susceptibility and Pathogen-Specific Risk Factors for Udder Pathogens from Clinical Mastitis in Dairy Cows
}

\author{
Anna Duse, Karin Persson-Waller and Karl Pedersen * (D)
}

check for

updates

Citation: Duse, A.; Persson-Waller, K.; Pedersen, K. Microbial Aetiology, Antibiotic Susceptibility and Pathogen-Specific Risk Factors for Udder Pathogens from Clinical Mastitis in Dairy Cows. Animals 2021, 11, 2113. https://doi.org/10.3390/ ani11072113

Academic Editor: Alfonso Zecconi

Received: 1 July 2021

Accepted: 14 July 2021

Published: 16 July 2021

Publisher's Note: MDPI stays neutral with regard to jurisdictional claims in published maps and institutional affiliations.

Copyright: (c) 2021 by the authors. Licensee MDPI, Basel, Switzerland. This article is an open access article distributed under the terms and conditions of the Creative Commons Attribution (CC BY) license (https:// creativecommons.org/licenses/by/ $4.0 /)$.
Department of Animal Health and Antimicrobial Strategies, National Veterinary Institute 75189 Uppsala, Sweden; anna.duse@sva.se (A.D.); karin.persson-waller@sva.se (K.P.-W.)

* Correspondence: karl.pedersen@sva.se; Tel.: +46-18-674288

Simple Summary: Mastitis is among the diseases in dairy cows that most often require antibiotic treatment. In order to maintain optimal treatment, it is important to have updated knowledge about the causative agents and their antibiotic resistance patterns. This investigation aimed to reveal the most important bacterial pathogens and their resistance patterns in Sweden, and we also identified some risk factors for infection with certain pathogens. The bacteria that were the most common causes of mastitis were, in descending order, Staphylococcus aureus, Streptococcus dysgalactiae, Escherichia coli, and Streptococcus uberis. Only a few Gram-positive bacteria were resistant to penicillin, and in general, the occurrence of antibiotic resistance was low. Therefore, the potential for antibiotic treatment of bovine mastitis in Sweden is good.

Abstract: Mastitis is one of the most important infectious diseases and one of the diseases that causes the greatest use of antibiotics in dairy cows. Therefore, updated information on the bacteria that cause mastitis and their antibiotic susceptibility properties is important. Here, for the first time in over 10 years, we updated the bacterial findings in clinical mastitis in Swedish dairy cows together with their antibiotic resistance patterns and risk factors for each bacterial species. During the period 2013-2018, samples from clinical mastitis were collected, together with information on the cows and herds of origin. The samples were cultured, and a total of 664 recovered bacterial isolates were subjected to susceptibility testing. Staphylococcus aureus (S. aureus) was the most common pathogen and accounted for $27.8 \%$ of diagnoses, followed by Streptococcus dysgalactiae (S. dysgalactiae) (15.8\%), Escherichia coli (E. coli) (15.1\%), Streptococcus uberis (S. uberis) (11.4\%), Trueperella pyogenes (T. pyogenes) (7.7\%), non-aureus staphylococci (NAS) (2.8\%), Klebsiella spp. (2.7\%), Enterococcus spp. $(1.3 \%)$, and Streptococcus agalactiae (S. agalactiae) (1.2\%). Various other bacteria accounted for $2.6 \%$. Staphylococci were, in general, susceptible to most antibiotics, but $2.6 \%$ of $S$. aureus and $30.4 \%$ of NAS were resistant to penicillin. No methicillin-resistant staphylococci were found. All S. agalactiae were susceptible to penicillin. Bimodal and trimodal MIC distributions for penicillin in S. dysgalactiae and S. uberis, respectively, indicate acquired reduced susceptibility in some isolates. The mostly unimodal MIC distributions of T. pyogenes indicate that acquired resistance does usually not occur in this species. Among E. coli, $14.7 \%$ were resistant to at least one antibiotic, most often ampicillin $(8.7 \%)$, streptomycin $(7.8 \%)$, or sulphamethoxazole $(6.9 \%)$. Klebsiella spp. had low resistance to tetracycline $(9.1 \%)$ but is considered intrinsically resistant to ampicillin. Pathogen-specific risk factors were investigated using multivariable models. Staphylococcus aureus, S. dysgalactiae, and T. pyogenes were more common, while E. coli was less common in quarters with more than one pathogen. $S$. aureus and T. pyogenes were mostly seen in early lactation, while E. coli was more common in peak to mid lactation and S. dysgalactiae in early to peak lactation. Trueperella pyogenes and Klebsiella spp. were associated with a previous case of clinical mastitis in the current lactation. Staphylococcus aureus was associated with tie stalls and T. pyogenes with loose housing. All pathogens except E. coli and S. dysgalactiae had a seasonal distribution. In conclusion, the aetiological agents for clinical bovine mastitis have remained relatively stable over the last 10-15 years, S. aureus, S. dysgalactiae, E. coli and S. uberis being the most important. Resistance to penicillin among Gram-positive agents was low, 
and in general, antibiotic resistance to other compounds was low among both Gram-positive and Gram-negative agents.

Keywords: mastitis; antibiotic resistance; dairy cow; risk factors; Sweden

\section{Introduction}

Globally, mastitis is one of the most important infectious diseases in dairy herds [1] The majority of clinical cases are caused by a limited number of specific pathogens, but in fact, a very large array of bacterial species may infect the udder. The panorama can also vary between countries and regions within each country. In order to implement suitable strategies against mastitis, it is important to understand the panorama of udder pathogens and to monitor trends over time. Mastitis is also by far the most important reason for antibiotic treatment in Swedish dairy herds, and about $60 \%$ of all antibiotics prescribed for parenteral treatment of dairy cows are for mastitis [2]. Hence, sustaining the efficacy of antibiotics is very important for dairy cow welfare and herd economics. Acquired antibiotic resistance in bacteria is, however, an increasing threat, and surveillance of antibiotic susceptibility of bacteria, including mastitis-causing pathogens, is recommended by the World Organisation for Animal Health (OIE) and several other organisations [3,4]. Results of such monitoring will guide therapeutic decisions and show possible trends, indicating a possible need for interventions regarding antibiotic use [4].

In Swedish dairy cattle herds, benzylpenicillin is by far the most prescribed antibiotic and drug-of-choice for most Gram-positive udder pathogens. Swedish veterinarians have, for several decades, complied with these recommendations, and it has been shown that benzylpenicillin, administered systemically in accordance with distributers' recommendations, is used in over $80 \%$ of treatments for mastitis [2,5]. Although this antibiotic has a narrow spectrum, use of it may still enforce a selective pressure on certain bacterial species, which can increase the prevalence of antibiotic resistance [6]. Regular monitoring of the spectrum and susceptibility of udder pathogens is therefore warranted. The most recent nation-wide surveys were performed in 1994-1995 and 2002-2003 [7-9], and a timely update was therefore deemed necessary.

In the survey from 2002-2003, agent-specific risk factors were also sought [9]. Since the dairy herd structure has changed since then, with larger herds, more loose housing as opposed to tie stalls, and more cows milked in automatic milking systems, etc., it is possible that the importance of certain risk factors may have changed. Such information may be useful in future strategies combatting mastitis and is therefore of value to monitor.

The objectives of this study were to reveal the microbial panorama of udder pathogens associated with clinical mastitis and their antibiotic susceptibility in Swedish dairy cows and compare the results with previous investigations to identify important developments. Another objective was to investigate pathogen-specific risk factors.

\section{Materials and Methods}

\subsection{Sampling}

In August 2013, a project was initiated to continuously monitor the aetiology and antibiotic resistance in acute clinical mastitis in Swedish dairy cows. The project is a collaboration between the Swedish National Veterinary Institute (SVA) and Farm and Animal Health AB. Thirty-eight veterinary practices distributed throughout Sweden in the District Veterinary Organization collected milk samples. In Sweden, only veterinarians can prescribe antibiotics, and the veterinarian must visit the sick animal and make a diagnosis before prescribing such drugs. Each veterinary practice was encouraged to submit the first cases of clinical mastitis they diagnosed every month. This was to avoid selected cases being sampled for laboratory investigation. Only one quarter should be sampled. Samples should be taken from a lactating cow with observable abnormalities in milk in at least one 
quarter, with or without signs of swelling of the mammary gland or systemic illness. If more than one quarter was affected, only the most severely affected quarter should be sampled. When a case was identified, the responsible veterinarian collected individual milk samples aseptically from the selected quarter. At sampling, the following data about the cow and the herd were also registered: breed of the cow, udder quarter, lactation number, udder disease score based on cow somatic cell count (SCC) at the last three milk recordings [10], the result of California Mastitis Test (CMT; scored $1-5$ where $1=$ negative and $5=$ strong gel formation), date of latest calving, if the cow had experienced another case of clinical mastitis during the current lactation, if she had been treated with antibiotics during the preceding 30 days or at drying off, the main housing system (tie-up or loose-housing) used in the herd, and if automatic milking systems (AMS) were used. Samples were collected from August 2013 to December 2018. The geographical location of the farms was defined from their regional Nomenclature des Unités Territoriales Statistiques (NUTS) location [11].

\subsection{Culture of Samples}

Storage and shipping of milk samples was carried out according to regular routines of the District Veterinary Organization. The samples, collected in sterile plastic tubes, were kept chilled from time of sampling to shipping and were sent at ambient temperature by over-night postal mail to SVA, Uppsala, Sweden on the day of collection. At SVA, bacteriological analysis was performed according to accredited routines (EN/ISO 17023). Briefly, $10 \mu \mathrm{L}$ were streaked onto blood agar ( $5 \%$ bovine blood) with esculin, incubated at 37 ${ }^{\circ} \mathrm{C}$, and evaluated after 18-24 and 36-48 h. Growth on agar plates was initially characterized on the basis of colony morphology and $\alpha-, \beta-$, or double haemolysis. Colonies of potential udder pathogens, except those considered as $S$. aureus based on colony morphology and double haemolysis, were put forward for genus and species identification with matrixassisted laser desorption/ionization-time of flight mass spectrometry (MALDI-TOF MS) on a MALDI Biotyper (Bruker Daltonics, Bremen, Germany), using methods and spectra scores as previously described [12].

Evaluation of growth was performed according to accredited routines (EN/ISO 17023) at SVA. A milk sample was considered positive if at least one colony-forming unit (CFU) of Staphylococcus aureus (S. aureus), non-aureus staphylococci (NAS), Streptococcus agalactiae (S. agalactiae), Streptococcus dysgalactiae (S. dysgalactiae), Streptococcus uberis (S. uberis), Streptococcus parauberis (S. parauberis), Trueperella pyogenes (T. pyogenes), Pasteurella/Mannheimia spp., Escherichia coli (E. coli), Klebsiella spp., or yeast was detected. For other bacteria, a positive classification required $\geq 5 \mathrm{CFU}$. If the growth of 2 colony types was detected, the milk sample was classified as having a mixed infection. If $\geq 3$ colony types were detected, the milk sample was classified as contaminated. However, in line with National Mastitis Council (NMC) guidelines (2017), if low-level contamination, e.g., a few colonies of different morphologies, were found together with numerous colonies of one of the pathogens mentioned above, the sample was diagnosed as positive for the specific pathogen. Moreover, if growth of S. aureus, S. dysgalactiae and T. pyogenes was found in the same milk sample, the sample was diagnosed as positive for all three pathogens. This was in line with the evaluation used by Ericsson-Unnerstad et al. [9] and the laboratory routine (Table 1). 
Table 1. Distribution of 823 microbial diagnoses from 755 udder quarters from cows with clinical mastitis (per cent, $95 \%$ confidence interval (CI) and number of diagnoses).

\begin{tabular}{|c|c|c|c|c|c|c|c|}
\hline \multirow[t]{2}{*}{ Diagnosis } & \multicolumn{3}{|c|}{$\begin{array}{c}2013-2018 \\
(n=823)\end{array}$} & \multicolumn{2}{|c|}{$\begin{array}{c}2002-2003 \\
(n=1056) \\
\text { Ericsson Unnerstad } \\
\text { et al. 2009 [9] }\end{array}$} & \multicolumn{2}{|c|}{$\begin{array}{c}1994-1995 \\
(n=837) \\
\text { Nilsson et al. } \\
1997[7]\end{array}$} \\
\hline & $n$ & $\%$ & $\mathrm{CI}$ & $n$ & $\%$ & $n$ & $\%$ \\
\hline Staphylococcus aureus & 229 & 27.8 & $24.7-31.0$ & 225 & 21.3 & 181 & 21.6 \\
\hline Non-aureus staphylococci ${ }^{1}$ & 23 & 2.8 & $1.8-4.2$ & 65 & 6.2 & 35 & 4.2 \\
\hline Streptococcus dysgalactiae & 130 & 15.8 & $13.4-18.5$ & 165 & 15.6 & 113 & 13.5 \\
\hline Streptococcus uberis & 94 & 11.4 & $9.3-13.8$ & 117 & 11.1 & 128 & 15.3 \\
\hline Streptococcus agalactiae & 10 & 1.2 & $0.6-2.2$ & 6 & 0.6 & & \\
\hline Other streptococci & 4 & 0.5 & $0.1-1.2$ & 9 & 0.9 & 11 & 1.2 \\
\hline Enterococci & 11 & 1.3 & $0.7-2.4$ & 8 & 0.8 & 7 & 0.8 \\
\hline Trueperella pyogenes & 63 & 7.7 & $5.9-9.7$ & 64 & 6.1 & 77 & 9.2 \\
\hline Escherichia coli & 124 & 15.1 & $12.7-17.7$ & 168 & 15.9 & 134 & 16.0 \\
\hline Klebsiella spp. & 22 & 2.7 & $1.7-4.0$ & 44 & 4.2 & 19 & 2.2 \\
\hline Other Enterobacterales 2 & 12 & 1.5 & $0.8-2.5$ & $9^{1}$ & 0.9 & 8 & 0.8 \\
\hline Other bacteria ${ }^{3}$ & 21 & 2.6 & $1.6-3.9$ & $16^{2}$ & 1.5 & 9 & 0.9 \\
\hline Contaminated & 40 & 4.9 & $3.5-6.6$ & 48 & 4.5 & 49 & 5.9 \\
\hline No growth & 40 & 4.9 & $3.5-6.6$ & 112 & 10.6 & 66 & 7.9 \\
\hline
\end{tabular}

${ }^{1}$ S. chromogenes (5), S. epidermidis (5), S. haemolyticus (5), S. simulans (3), S. cohnii (2), S. hyicus (1), un-identified (2). ${ }^{2}$ Enterobacter spp. (4), Citrobacter freundii (2), Serratia spp. (3), Proteus mirabilis (2), Yersinia (1). ${ }^{3}$ Bacillus spp. (3), Helcococcus spp. (3), Lactococcus spp. (2), Pasteurella multocida (2) Corynebacterium spp. (1), yeast (6), Mannheimia haemolytica (2), Acinetobacter spp. (1), Gram-positive rod (1).

\subsection{Antimicrobial Susceptibility Testing}

Isolates of S. aureus, NAS, S. uberis, S. dysgalactiae, S. agalactiae, T. pyogenes, E. coli and Klebsiella spp. were subjected to susceptibility testing directly upon isolation by determination of minimum inhibitory concentration (MIC) using broth microdilution. Testing was performed essentially according to the recommendations of the Clinical and Laboratory Standards Institute [13] using VetMIC ${ }^{\mathrm{TM}}$ panels (National Veterinary Institute, Uppsala, Sweden) and cation-adjusted Mueller-Hinton broth (Becton Dickinson, Cockeysville, USA). The following modifications from CLSI were made: for the inoculum, the broth culture method was used for the testing of non-fastidious bacteria. Colony material was transferred into $5 \mathrm{~mL}$ of Mueller-Hinton broth and incubated at $35^{\circ} \mathrm{C}$ for $3-5 \mathrm{~h}$, after which $3-10 \mu \mathrm{L}$ was transferred into $10 \mathrm{~mL}$ of Mueller-Hinton broth, which was subsequently used to inoculate the microtiter plates, with $50 \mu \mathrm{L}$ in each well. For T. pyogenes, the direct colony suspension method was used. However, the inoculum was prepared using cation-adjusted Mueller-Hinton broth supplemented with $5 \%$ horse serum. The MIC panels were incubated at $35^{\circ} \mathrm{C}$, and T. pyogenes was read after $48 \mathrm{~h}$, whereas other bacteria were read after $16-18 \mathrm{~h}$. Antibiotics and test ranges are indicated in Tables $2-5$. For oxacillin susceptibility testing of staphylococci, the broth was supplemented with $2 \% \mathrm{NaCl}$. Staphylococcus aureus ATCC 29,213 , S. aureus ATCC 25,923 and E. coli ATCC 25,922 were used as quality control reference strains. Staphylococci were examined for $\beta$-lactamase production using the "clover-leaf" method [14]. Isolates with MIC $>2 \mathrm{mg} / \mathrm{L}$ for oxacillin or MIC $>4 \mathrm{mg} / \mathrm{L}$ for cefoxitin were confirmed as methicillin-resistant staphylococci (MRS) through detection of the mecA or $m e c C$ genes, applying real-time PCR as previously described [15].

Isolates of Enterobacterales with cefotaxime or ceftazidime MIC above the respective EUCAST cut-off value, which depends on the species, were further analyzed with phenotypic confirmatory tests for production of extended spectrum beta-lactamases (ESBLs). The tests were performed with and without clavulanic acid in Sensititre EUVSEC2 microdilution panels (ThermoFisher, Oakwood Village, OH, USA) and interpreted according to the European Committee on Antimicrobial Susceptibility Testing (EUCAST) (http \www.eucast.org, accessed on 14 July 2021). None of the isolates had a phenotype indicative of ESBL production, and further genotypic testing was not necessary. 
Isolates were classified as susceptible (wildtype) or resistant (non-wildtype) based on EUCAST epidemiological cut-off values (ECOFFs). For E. coli, the ECOFF for trimethoprimsulphadoxazole $(>0.25 \mathrm{mg} / \mathrm{L})$ could not be used since it was outside the range of tested concentrations. The same applied to the cut-off value for fusidic acid in S. agalactiae (>32 mg/L). For Klebsiella spp., the cut-off value for cefotaxime $(>0.125 \mathrm{mg} / \mathrm{L})$ would have falsely categorized one K. pneumonia isolate (cut-off $>0.25 \mathrm{mg} / \mathrm{L}$ ) as non-wild-type, and hence the cut-off for K. pneumonia was used. The same applied to enrofloxacin, colistin, and tetracycline. The cut off-values used are provided in Tables $2-5$. Staphylococci were considered penicillin susceptible or resistant on the basis of the production of $\beta$-lactamase. When EUCAST ECOFFs were not available, isolates were not classified.

Isolates of E. coli with ciprofloxacin MIC $>0.06 \mathrm{mg} / \mathrm{L}$ and nalidixic acid MIC $<16 \mathrm{mg} / \mathrm{L}$ were selected for PCR detection of plasmid-mediated quinolone resistance genes, including $q n r \mathrm{~A}, q n r \mathrm{~B}, q n r \mathrm{~S}$, and $a a c\left(6^{\prime}\right)-1 \mathrm{~b}-\mathrm{cr}$, using the PCR assays described earlier [16].

Table 2. Number of isolates (No), resistance (per cent, 95\% CI in brackets), and MIC distribution (\%) for Staphylococcus aureus $(n=227)$ and non-aureus staphylococci (NAS) $(n=21)$ from clinical mastitis in dairy cows. White fields indicate test range for each antibiotic.

\begin{tabular}{|c|c|c|c|c|c|c|c|c|c|c|c|c|c|c|c|}
\hline \multirow{2}{*}{ Substance } & \multirow{2}{*}{ Species } & \multirow{2}{*}{ No } & \multirow{2}{*}{$\begin{array}{c}\text { Resistance } \\
(\%)\end{array}$} & \multicolumn{12}{|c|}{ Distribution (\%) of MICs (mg/L) } \\
\hline & & & & $\leq \mathbf{0 . 0 3}$ & 0.06 & 0.12 & 0.25 & 0.5 & 1 & 2 & 4 & 8 & 16 & $32 \quad 64$ & $>64$ \\
\hline \multirow{2}{*}{ Cefoxitin } & S. aureus & 97 & $0(0.0-3.7)$ & & & & & & 1.0 & 49.5 & 49.5 & & & & \\
\hline & NAS & 10 & 0 & & & & 10.0 & & & 80.0 & 10.0 & & & & \\
\hline \multirow{2}{*}{ Cephalothin } & S. aureus & 227 & $0(0.0-1.6)$ & & & 51.5 & 44.5 & 3.5 & 0.4 & & & & & & \\
\hline & NAS & 21 & - & & & 47.6 & 38.1 & 4.8 & 9.5 & & & & & & \\
\hline \multirow{2}{*}{ Chloramphenicol } & S. aureus & 227 & $0(0.0-1.6)$ & & & & & & & & 19.8 & 77.1 & 3.1 & & \\
\hline & NAS & 21 & $0(0.0-16.1)$ & & & & & & & 4.8 & 71.4 & 19.1 & 4.8 & & \\
\hline \multirow{2}{*}{ Ciprofloxacin } & S. aureus & 227 & $0(0.0-1.6)$ & & & 21.6 & 55.1 & 22.5 & 0.9 & & & & & & \\
\hline & NAS & 21 & $0(0.0-16.1)$ & & & 57.1 & 38.1 & 4.8 & & & & & & & \\
\hline \multirow{2}{*}{ Clindamycin } & S. aureus & 227 & $0.9(0.1-3.1)$ & & & & 99.1 & 0.9 & & & & & & & \\
\hline & NAS & 21 & $14.3(5.0-34.6)$ & & & & 85.7 & 4.8 & 4.8 & 4.8 & & & & & \\
\hline \multirow[b]{2}{*}{ Enrofloxacin } & S. aureus & 97 & - & & & 46.4 & 49.5 & 4.1 & & & & & & & \\
\hline & NAS & 10 & - & & & 70.0 & 30.0 & & & & & & & & \\
\hline \multirow{2}{*}{ Erythromycin } & S. aureus & 227 & $0(0.0-1.6)$ & & & & 56.0 & 34.8 & 9.2 & & & & & & \\
\hline & NAS & 21 & $9.5(2.7-28.9)$ & & & & 71.4 & 19.1 & & & & 4.8 & & 4.8 & \\
\hline \multirow{2}{*}{ Fusidic acid } & S. aureus & 227 & $4.4(2.1-8.0)$ & & & & 73.1 & 22.5 & 2.6 & 0.9 & 0.4 & 0.4 & & & \\
\hline & NAS & 21 & $14.3(3.0-36.3)$ & & & & 57.1 & 23.8 & 4.8 & & 4.8 & 9.6 & & & \\
\hline \multirow[b]{2}{*}{ Gentamicin } & S. aureus & 227 & $0(0.0-1.6)$ & & & & & 89.4 & 9.3 & 1.3 & & & & & \\
\hline & NAS & 21 & $0(0.0-16.1)$ & & & & & 100.0 & & & & & & & \\
\hline \multirow{2}{*}{ Kanamycin } & S. aureus & 130 & $0.8(0.0-4.2)$ & & & & 0.8 & 9.2 & 16.9 & 54.6 & 10.8 & 6.9 & 0.8 & & \\
\hline & NAS & 11 & - & & & & 63.6 & 27.3 & 9.1 & & & & & & \\
\hline \multirow{2}{*}{ Linezolid } & S. aureus & 62 & $0.0(0.0-5.8)$ & & & & & & 14.5 & 82.3 & 3.2 & & & & \\
\hline & NAS & 5 & $0.0(0.0-52.2)$ & & & & & & 60.0 & 40.0 & & & & & \\
\hline \multirow{2}{*}{ Oxacillin } & S. aureus & 165 & $0.6(0.0-3.3)$ & & & & 45.5 & 23.6 & 25.5 & 4.9 & $0.6^{1}$ & & & & \\
\hline & NAS & 16 & $6.2(0.2-30.2)$ & & & & 50.0 & 25.0 & 18.8 & & $6.2^{1}$ & & & & \\
\hline \multirow{2}{*}{ Penicillin $^{2}$} & S. aureus & 227 & $2.6(1.0-5.7)$ & 60.4 & 32.2 & 4.0 & 0.4 & 0.4 & 0.4 & 0.9 & 0.9 & 0.4 & & & \\
\hline & NAS & 23 & $30.4(13.2-52.9)$ & 33.3 & 28.6 & & 9.5 & 9.5 & 9.5 & & 9.5 & & & & \\
\hline \multirow{2}{*}{ Tetracycline } & S. aureus & 227 & $0.9(0.1-3.1)$ & & & & & 96.5 & 2.6 & 0.9 & & & & & \\
\hline & NAS & 21 & $0(0.0-16.1)$ & & & & & 95.2 & 4.8 & & & & & & \\
\hline \multirow{2}{*}{ Trimethoprim } & S. aureus & 227 & $8.8(5.5-13.3)$ & & & & & 20.7 & 41.0 & 29.5 & 7.5 & 1.3 & & & \\
\hline & NAS & 21 & - & & & & & 28.6 & 14.3 & 19.1 & 19.1 & 9.5 & 9.5 & & \\
\hline Trimethoprim- & S. aureus & 97 & $0(0.0-3.7)$ & & & 97.9 & 2.1 & & & & & & & & \\
\hline Sulphadoxazole & NAS & 10 & - & & & 60.0 & 20.0 & 10.0 & 10.0 & & & & & & \\
\hline
\end{tabular}

${ }^{1} 1$ S. aureus and 1 NAS tested negative for the mec $A$ and $m e c C$ gene. ${ }^{2}$ No cut-off value given, classification according to beta-lactamase production. 
Table 3. Number of tested isolates (No), resistance (per cent, $95 \%$ CI in brackets), and MIC distribution (\%) for Streptococcus agalactiae, Streptococcus dysgalactiae and Streptococcus uberis from clinical mastitis in dairy cows. White fields indicate test range for each antibiotic.

\begin{tabular}{|c|c|c|c|c|c|c|c|c|c|c|c|c|c|c|c|c|}
\hline \multirow{2}{*}{ Substance } & \multirow{2}{*}{ Species } & \multirow{2}{*}{ No } & \multirow{2}{*}{$\begin{array}{l}\text { Resistance } \\
(\%)\end{array}$} & \multicolumn{13}{|c|}{ Distribution (\%) of MICs $(\mu \mathrm{g} / \mathrm{L})$} \\
\hline & & & & $\leq 0.03$ & $\leq 0.06$ & 0.12 & 0.25 & 0.5 & 1 & 2 & 4 & 8 & 16 & 32 & 64 & $>64$ \\
\hline \multirow{3}{*}{ Cefoxitin } & S. agal. & 2 & - & & & & & & & 100.0 & & & & & & \\
\hline & S. dysg. & 41 & - & & & & 46.3 & 43.9 & 7.3 & 2.4 & & & & & & \\
\hline & S. uberis & 38 & - & & & & 2.6 & 47.4 & 26.3 & 7.9 & 10.5 & 5.3 & & & & \\
\hline \multirow{3}{*}{ Cephalothin } & S. agal. & 9 & - & & & 100.0 & & & & & & & & & & \\
\hline & S. dysg. & 120 & - & & & 96.7 & 1.7 & 0.8 & 0.8 & & & & & & & \\
\hline & S. uberis & 89 & - & & & 74.2 & 12.4 & 11.2 & 2.3 & & & & & & & \\
\hline \multirow{3}{*}{ Chloramphenicol } & S. agal. & 9 & - & & & & & & & 100.0 & & & & & & \\
\hline & S. dysg. & 120 & - & & & & & 1.7 & 25.0 & 65.0 & 7.5 & 0.8 & & & & \\
\hline & S. uberis & 89 & - & & & & & & 4.5 & 32.6 & 61.8 & 1.1 & & & & \\
\hline \multirow{3}{*}{ Ciprofloxacin } & S. agal. & 9 & $0(0.0-33.6)$ & & & & & 77.8 & 22.2 & & & & & & & \\
\hline & S. dysg. & 120 & - & & & & 17.5 & 72.5 & 9.2 & 0.8 & & & & & & \\
\hline & S. uberis & 89 & - & & & 1.1 & 20.2 & 52.8 & 25.8 & & & & & & & \\
\hline \multirow{3}{*}{ Clindamycin } & S. agal. & 9 & $0(0.0-33.6)$ & & & & 100.0 & & & & & & & & & \\
\hline & S. dysg. & 120 & $0(0.0-3.0)$ & & & & 100.0 & & & & & & & & & \\
\hline & S. uberis & 89 & - & & & & 96.6 & 1.1 & 1.1 & & & & & & 1.1 & \\
\hline \multirow{3}{*}{ Enrofloxacin } & S. agal. & 2 & - & & & & & 50.0 & 50.0 & & & & & & & \\
\hline & S. dysg. & 41 & - & & & 2.4 & 9.8 & 85.4 & 2.4 & & & & & & & \\
\hline & S. uberis & 38 & - & & & & 15.8 & 68.4 & 15.8 & & & & & & & \\
\hline \multirow{3}{*}{ Erythromycin } & S. agal. & 9 & $0(0-33.6)$ & & & & 100.0 & & & & & & & & & \\
\hline & S. dysg. & 120 & - & & & & 99.2 & & & 0.8 & & & & & & \\
\hline & S. uberis & 89 & - & & & & 96.7 & 1.1 & & & & 1.1 & & 1.1 & & \\
\hline \multirow{3}{*}{$>$ Fusidic acid } & S. agal. & 9 & - & & & & & & & & 22.2 & 78.8 & & & & \\
\hline & S. dysg. & 120 & - & & & 0.9 & & 3.3 & 47.5 & 0.8 & 5.8 & 1.7 & & & & \\
\hline & S. uberis & 89 & - & & & & & 1.1 & 1.1 & 23.6 & 51.7 & 22.4 & & & & \\
\hline \multirow{3}{*}{ Gentamicin } & S. agal. & 9 & - & & & & & & & 44.4 & 44.4 & 11.1 & & & & \\
\hline & S. dysg. & 120 & - & & & & & 43.4 & 40.0 & 11.7 & 2.5 & 0.8 & 0.8 & 0.8 & & \\
\hline & S. uberis & 89 & - & & & & & 21.4 & 6.7 & 19.1 & 27.0 & 15.7 & 9.0 & 1.1 & & \\
\hline \multirow{3}{*}{ Kanamycin } & S. agal. & 7 & - & & & & & & & & & & 14.3 & 57.1 & 28.6 & \\
\hline & S. dysg. & 79 & - & & & & & 6.3 & 2.5 & 22.8 & 20.4 & 25.3 & 7.6 & 5.1 & & \\
\hline & S. uberis & 51 & - & & & & 5.9 & 9.8 & & 7.8 & 5.9 & 27.5 & 29.4 & 11.8 & 1.9 & \\
\hline \multirow{3}{*}{ Linezolid } & S. agal. & 0 & - & & & & & & & & & & & & & \\
\hline & S. dysg. & 24 & - & & & & & & 100.0 & & & & & & & \\
\hline & S. uberis & 24 & - & & & & & & 50.0 & 50.0 & & & & & & \\
\hline & S. agal. & 9 & - & & & & 66.7 & 22.2 & 11.1 & & & & & & & \\
\hline Oxacillin & S. dysg. & 96 & - & & & & 95.9 & 1.0 & 2.1 & & 1.0 & & & & & \\
\hline & S. uberis & 65 & - & & & & 86.1 & 1.5 & 3.1 & 6.2 & 3.1 & & & & & \\
\hline & S. agal. & 9 & $0(0.0-33.6)$ & 44.4 & 44.4 & 11.1 & & & & & & & & & & \\
\hline Penicillin & S. dysg. & 120 & - & 97.5 & 1.7 & & 0.9 & & & & & & & & & \\
\hline & S. uberis & 89 & - & 75.3 & 5.6 & 16.9 & 1.1 & & & & 1.1 & & & & & \\
\hline & S. agal. & 9 & $22.2(2.8-60.0)$ & & & & & 77.8 & & & & 11.1 & & & 11.1 & \\
\hline Tetracycline & S. dysg. & 120 & - & & & & & 8.4 & 10.0 & 49.2 & 27.5 & 2.5 & 0.8 & 0.8 & & 0.8 \\
\hline & S. uberis & 89 & - & & & & & 95.5 & 2.3 & & & 1.1 & & & 1.1 & \\
\hline & S. agal. & 9 & - & & & & & & & 33.3 & 55.6 & 11.1 & & & & \\
\hline Trimethoprim & S. dysg. & 120 & - & & & & & 24.2 & 48.3 & 22.5 & 4.2 & & 0.8 & & & \\
\hline & S. uberis & 89 & - & & & & & 10.1 & 61.8 & 23.6 & 4.5 & & & & & \\
\hline & S. agal. & 2 & - & & & 50.0 & 50.0 & & & & & & & & & \\
\hline Trimethoprim- & S. dysg. & 41 & - & & & 85.4 & 14.6 & & & & & & & & & \\
\hline Sulphadoxazole & S. uberis & 38 & - & & & 78.9 & 21.1 & & & & & & & & & \\
\hline
\end{tabular}

\subsection{Risk Factor Analyses}

For the risk factor analyses, we excluded samples with no growth $(n=40)$, contaminated samples $(n=40)$, and samples without herd identity $(n=9)$. Quarter-level data were summarized at the cow level, yielding a 2-level dataset based on 755 udder quarter samples, 653 cows and 350 herds. A new variable, "number of diagnoses per quarter", was generated, describing quarter-level features.

Descriptive statistics of all herd variables are given in Supplementary Table S1. To reduce the amount of missing data for the variables housing and AMS, loose housing was assumed in herds with AMS, and herds with tie stalls were assumed not to have AMS. The continuous variable, "days in milk" (DIM), was not linearly related to the log of the outcome (probability of isolating a certain pathogen) and was therefore categorized in terms of early (<50 DIM), peak (50-109 DIM), mid (110-209 DIM) and late lactation (>209 DIM). The odds of isolation of a certain pathogen from a sample, given the presence/absence of different risk factors, were estimated using logistic regression. The dependent variables in each model determined whether the cow was a case $(1=$ yes $)$ or not $(0=$ no $)$ with respect to S. aureus, NAS, S. dysgalactiae, S. uberis, E. coli, Klebsiella spp., or T. pyogenes, respectively, defining seven different mastitis outcomes. Each cow was represented once in each model, 
either as a case or a non-case. If more than one bacterial species was isolated, the cow was considered a case for each species and otherwise as a control. Non-cases in each model were cows with clinical mastitis caused by pathogens other than the one of interest.

Table 4. Number of tested isolates (No), resistance (per cent, 95\% CI in brackets), and MIC distribution (\%) for Escherichia coli and Klebsiella spp. isolated from cases of clinical mastitis in dairy cows. White fields indicate test range for each antibiotic.

\begin{tabular}{|c|c|c|c|c|c|c|c|c|c|c|c|c|c|c|c|c|c|c|c|c|c|}
\hline \multirow{2}{*}{ Substance } & \multirow{2}{*}{ Species } & \multirow{2}{*}{ No } & \multirow{2}{*}{$\begin{array}{c}\text { Resistance } \\
\quad(\%)\end{array}$} & \multicolumn{18}{|c|}{ Distribution (\%) of MICs (mg/L) } \\
\hline & & & & $\leq 0.016$ & 0.03 & 0.06 & 0.12 & 0.25 & 0.5 & 1 & 2 & 4 & 8 & 16 & 32 & 64 & 128 & 256 & 512 & 1024 & $>1024$ \\
\hline \multirow[t]{2}{*}{ Ampicillin } & E. coli & 116 & $8.6(4.2-15.3)$ & & & & & & & 17.2 & 58.6 & 14.7 & 0.9 & & 0.9 & & 0.9 & 6.9 & & & \\
\hline & Klebsiella & 22 & $95.4(77.2-99.9)$ & & & & & & & & & & 4.6 & 18.1 & 45.4 & 22.7 & 4.6 & 4.6 & & & \\
\hline Ceftazidime & $\begin{array}{c}\text { E. coli } \\
\text { Klebsiella }\end{array}$ & $\begin{array}{l}116 \\
22\end{array}$ & $\begin{array}{l}4.3(1.4-9.8) \\
0(0.0-15.4)\end{array}$ & & & & & $\begin{array}{l}80.2 \\
81.8\end{array}$ & $\begin{array}{l}15.5 \\
18.2\end{array}$ & 3.5 & 0.9 & & & & & & & & & & \\
\hline \multirow{2}{*}{ Cefotaxime } & E. coli & 116 & $0(0.0-3.1)$ & & & 57.8 & 39.7 & 2.6 & & & & & & & & & & & & & \\
\hline & Klebsiella & 22 & $0(0.0-15.4)$ & & & 81.8 & 13.6 & 4.6 & & & & & & & & & & & & & \\
\hline Chloramphenicol & E. coli & 116 & $0(0.0-3.1)$ & & & & & & & & 7.8 & 57.8 & 34.5 & & 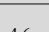 & & & & & & \\
\hline \multirow{2}{*}{ Ciprofloxacin } & $\frac{\text { Rlebsiella }}{\text { E. coli }}$ & $\frac{22}{116}$ & $\frac{-}{17(0,2-61)}$ & 10.3 & 69.8 & 18.1 & & & 0.9 & 0.9 & & & 4.6 & & 4.6 & & & & & & \\
\hline & Klebsiella & 22 & $4.6(0.1-22.8)$ & 4.6 & 31.8 & $\begin{array}{l}10.1 \\
36.3\end{array}$ & 22.7 & & 4.6 & & & & & & & & & & & & \\
\hline \multirow{2}{*}{ Colistin } & E. coli & 116 & $6.0(2.5-12.0)$ & & & & & & 25.0 & 42.2 & 26.7 & 6.0 & & & & & & & & & \\
\hline & Klebsiella & 22 & $4.6(0.1-22.8)$ & & & & & & 9.1 & 63.6 & 22.7 & 4.6 & & & & & & & & & \\
\hline \multirow{2}{*}{ Enrofloxacin } & E. coli & 28 & $0.0(0.0-12.3)$ & & & 100.0 & & & & & & & & & & & & & & & \\
\hline & Klebsiella & 6 & - & & & 100.0 & & & & & & & & & & & & & & & \\
\hline Florfenicol & E. coli & 116 & $0(0.0-3.1)$ & & & & & & & & & 41.4 & 53.5 & 5.2 & & & & & & & \\
\hline \multirow{2}{*}{ Kanamycin } & $\frac{\text { Klebsiella }}{\text { E. coli }}$ & $\frac{22}{88}$ & $\frac{-}{2.3(0.3-8.0)}$ & & & & & & & & & 68.2 & $\begin{array}{l}27.3 \\
97.7\end{array}$ & & $\begin{array}{l}4.5 \\
2.3\end{array}$ & & & & & & \\
\hline & Klebsiella & 16 & $2.0(0.0-0.0)$ & & & & & & & & & & 100.0 & & & & & & & & \\
\hline Gentamicin & $\begin{array}{l}\text { E. coli } \\
\text { Klebsiella }\end{array}$ & $\begin{array}{l}116 \\
22\end{array}$ & $\begin{array}{l}0.9(0.0-4.7) \\
0(00-15.4)\end{array}$ & & & & & & 79.3 & 18.1 & 1.7 & & & 0.9 & & & & & & & \\
\hline \multirow[b]{2}{*}{ Nalidixic acid } & $\begin{array}{l}\text { Klebsiella } \\
\text { E. coli }\end{array}$ & $\begin{array}{c}22 \\
116\end{array}$ & $\begin{array}{c}0(0.0-15.4) \\
0.9(0.0-4.7)\end{array}$ & & & & & & & & 47.4 & 47.4 & 4.3 & & & & & 0.9 & & & \\
\hline & Klebsiella & 22 & - & & & & & & & & 45.4 & 40.9 & 4.6 & 9.1 & & & & & & & \\
\hline \multirow{2}{*}{ Streptomycin } & E. coli & 116 & $7.8(3.6-14.2)$ & & & & & & & & & 50.9 & 37.9 & 3.5 & 0.9 & 2.6 & 4.3 & & & & \\
\hline & Klebsiella & 22 & - & & & & & & & & & 72.7 & 4.6 & 13.6 & 4.6 & 4.5 & & & & & \\
\hline \multirow{2}{*}{ Sulphamethoxazole } & E. coli & 116 & $6.9(3.0-13.1)$ & & & & & & & & & & 19.0 & 50.9 & 23.3 & & & & & & 6.9 \\
\hline & Klebsiella & 22 & & & & & & & & & & & 9.1 & 22.7 & 45.4 & 18.2 & & & & & 4.6 \\
\hline \multirow{2}{*}{ Tetracycline } & E. coli & 116 & $4.3(1.4-9.8)$ & & & & & & & 64.7 & 31.0 & & & & & 2.6 & 0.9 & 0.9 & & & \\
\hline & Klebsiella & 22 & $9.1(1.1-29.2)$ & & & & & & & 63.6 & 22.7 & 4.6 & & 4.6 & 4.5 & & & & & & \\
\hline \multirow{2}{*}{ Trimethoprim } & E. coli & 116 & $3.5(0.9-8.6)$ & & & & 12.1 & 39.7 & 39.7 & 5.2 & & & & & 3.5 & & & & & & \\
\hline & Klebsiella & 22 & - & & & & & 31.8 & 50.0 & 13.6 & & 4.6 & & & & & & & & & \\
\hline Trimethoprim- & E. coli & 28 & & & & & & & 92.9 & & & & 7.1 & & & & & & & & \\
\hline Sulphadoxazole & Klebsiella & 6 & $0.0(0.0-45.9)$ & & & & & & 100.0 & & & & & & & & & & & & \\
\hline
\end{tabular}

Table 5. Number of tested isolates (No) and MIC distribution (\%) for Trueperella pyogenes $(n=60)$ from clinical mastitis in dairy cows. White fields indicate test range for each antibiotic.

\begin{tabular}{|c|c|c|c|c|c|c|c|c|c|c|c|c|c|c|}
\hline \multirow{2}{*}{ Substance } & \multirow{2}{*}{ No } & \multicolumn{13}{|c|}{ Distribution (\%) of MICs ( $\mu \mathrm{g} / \mathrm{L})$} \\
\hline & & $\leq \mathbf{0 . 0 3}$ & 0.06 & 0.12 & 0.25 & 0.5 & 1 & 2 & 4 & 8 & 16 & 32 & 64 & $>64$ \\
\hline Cephalothin & 60 & & & 85.0 & 6.7 & 5.0 & 3.3 & & & & & & & \\
\hline Chloramphenicol & 60 & & & & & 25.0 & 48.3 & 26.7 & & & & & & \\
\hline Cefoxitin & 18 & & & & 100.0 & & & & & & & & & \\
\hline Ciprofloxacin & 60 & & & 5.0 & 6.7 & 5.0 & 65.0 & 18.3 & & & & & & \\
\hline Clindamycin & 60 & & & & 95.0 & 1.7 & 3.3 & & & & & & & \\
\hline Enrofloxacin & 18 & & & & & 5.6 & 88.8 & 5.6 & & & & & & \\
\hline Erythromycin & 60 & & & & 96.6 & 1.7 & 1.7 & & & & & & & \\
\hline Fusidic acid & 60 & & 21.7 & 23.3 & 35.0 & 18.3 & 1.7 & & & & & & & \\
\hline Gentamicin & 60 & & & & & 43.3 & 20.0 & 33.3 & 1.7 & 1.7 & & & & \\
\hline Kanamycin & 42 & & & & & 4.8 & 28.6 & 42.8 & 21.4 & 2.4 & & & & \\
\hline Linezolid & 11 & & & & & & 100.0 & & & & & & & \\
\hline Oxacillin & 49 & & & 14.2 & 20.5 & 16.3 & 18.4 & 14.3 & 12.2 & 4.1 & & & & \\
\hline Penicillin & 60 & 86.6 & 10.0 & 1.7 & 1.7 & & & & & & & & & \\
\hline Tetracycline & 60 & & & & & 86.6 & 5.0 & 1.7 & 1.7 & & 5.0 & & & \\
\hline Trimethoprim & 60 & & & & & 41.7 & 28.3 & 8.3 & 5.0 & 16.7 & & & & \\
\hline $\begin{array}{l}\text { Trimethoprim- } \\
\text { Sulphadoxazole }\end{array}$ & 17 & & & 11.8 & 11.8 & 29.4 & 5.9 & 5.9 & 35.4 & & & & & \\
\hline
\end{tabular}

There was a substantial number of missing values in the risk factor variables, ranging from 0 to $25 \%$ for different variables. Thus, each outcome was modelled twice (1) using only records with no missing data (complete case analysis, CC) and (2) using all records after multiple imputations of missing data (MI). Multiple imputation is a statistical technique for handling missing data. It has been shown multiple imputation analyses generally produce less biased results than complete case analyses [17]. Multiple imputation of missing values was carried out using the outcome variable and all risk factor variables, for which data were missing. Chained equations were used, and categorical predictors were imputed using a multinomial logit function, ordinal predictors using an ordered logit function and dichotomous predictors using a logit function. For each outcome, twenty imputed data sets 
were generated, and diagnostic plots comparing the distribution of the observed values with the imputed values were examined for the selected predictor variables.

Each herd was represented on average 1.86 times, hence assuming a limited clustering effect, and repeated observations within herd were accounted for using the cluster option (Stata). For each outcome and model (CC or MI), univariate associations (at $p<0.15)$ were used to identify candidate variables for the multivariable models. All two-way interactions between candidate predictors were included in the initial multivariable models and tested for significance. Non-significant ( $p>0.05$, likelihood ratio test) variables were eliminated in the multivariable analysis using a stepwise backward procedure. Model fit was assessed using the Hosmer and Lemeshow test, with data portioned in 10 deciles [18]. Stata, version 15 (StataCorp, College Station, TX, USA) was used for the statistical analyses.

\section{Results}

\subsection{Bacteriological Findings}

A total of 779 quarters from 738 cows were sampled. Thirteen samples had not been cultured, and the reason for not culturing was stated for two of them: delay in arrival to the laboratory. The remaining 766 udder quarter samples from 734 cows resulted in 835 microbial diagnoses (including no growth and contaminated samples). If a sample revealed growth of two pathogens, it was counted as two diagnoses. If the same bacterial species was diagnosed more than once from the same cow, only the isolate from the first sample was included. The other isolates $(n=12)$ were excluded due to risk of these being epidemiologically clustered. In total, 823 bacterial diagnoses from 755 udder quarters in 734 cows remained. One or more microbial species, based on culture and identification, were isolated from 675 (89\%) of the quarter samples. From 611 (90.5\%) of them, only one species was isolated; from 61 (8.9\%) samples, two species were isolated; and from $4(0.6 \%)$ samples, three species (S. aureus, S. dysgalactiae and T. pyogenes) were isolated. The distribution of the microbial diagnoses is shown in Table 1. For comparison, the results of two previous Swedish investigations are also presented $[7,9]$. Forty samples yielded no growth, while another 40 samples were contaminated. Staphylococcus aureus, S. dysgalactiae, E. coli and S. uberis were the most commonly detected species. Trueperella pyogenes, NAS, Klebsiella spp., Enterococcus spp., and S. agalactiae were found less often. Other bacteria were only found in a few cases each and together accounted for less than $1 \%$ each of the diagnoses.

\subsection{Antibiotic Susceptibility}

For the major mastitis-causing pathogens, antibiotic susceptibility results were available for 227 S. aureus isolates, 21 isolates of NAS, 120 S. dysgalactiae, 89 S. uberis, 9 S. agalactiae, 116 E. coli, 22 Klebsiella spp., and 60 T. pyogenes. Specific reasons why an isolate had not been tested or why susceptibility data were not in the laboratory database were not sought. For some antibiotics, susceptibility results were only available for a subset of the isolates due to changes in the composition of the susceptibility testing panels so that not all isolates were tested against all antibiotics depending on the antibiotics in the test panels. The results are provided as distributions of MICs, number of isolates tested and, when appropriate, cut-off values from EUCAST are available as percent resistant isolates in Tables 2-5. In the tables, white fields indicate the test range for each antibiotic. MICs above the range are shown as the concentration closest to the range. MICs equal to or lower than the lowest concentration tested are shown as the lowest tested concentration. Bold vertical lines indicate the ECOFFs when available.

Sixteen $(7.0 \%)$ of the 227 S. aureus isolates tested were resistant to one or more antibiotic. Penicillin resistance in S. aureus, i.e., $\beta$-lactamase production, occurred in six isolates $(2.6 \%)$ (Table 2). All these isolates had MICs for penicillin $>0.12 \mathrm{mg} / \mathrm{L}$. Five isolates $(2.2 \%)$ were resistant to more than one antibiotic: one isolate to penicillin and oxacillin, one isolate to clindamycin and fusidic acid, one isolate to fusidic acid and tetracycline, one isolate to penicillin and fusidic acid, and one isolate to fusidic acid, kanamycin, and tetracycline. Eleven $(52.3 \%)$ of the 21 NAS isolates tested were resistant to one or more antibiotic. 
Seven (30.4\%) of the 21 tested isolates were resistant to penicillin through $\beta$-lactamase production, and all of these, where MIC results were available, had MICs for penicillin > $0.12 \mathrm{mg} / \mathrm{L}$ (Table 2). Two isolates (9.5\%) were resistant to more than one antibiotic: one isolate to penicillin, clindamycin, fusidic acid, and erythromycin (not tested for oxacillin susceptibility) and one isolate to clindamycin, fusidic acid, erythromycin, and oxacillin. Bimodal distribution of MIC could be seen for cephalotin and trimethoprim. One isolate of S. aureus and one of NAS had MIC $>2 \mathrm{mg} / \mathrm{L}$ for oxacillin on testing at $37^{\circ} \mathrm{C}$. Neither the $S$. aureus nor the NAS isolate carried a mecA or a mecC gene, as confirmed by PCR.

Due to the lack of EUCAST cut-off values for streptococci in most antibiotics, the results are difficult to evaluate. Of the six S. agalactiae isolates, two were resistant to tetracycline (Table 3), for which one was also at the high end of the penicillin (MIC $=0.12 \mathrm{mg} / \mathrm{L}$ ) and oxacillin (MIC $=1 \mathrm{mg} / \mathrm{L}$ ) distributions. MICs to penicillin were below the epidemiological cutoff values in S. agalactiae. For S. dysgalactiae, there are no breakpoints except for clindamycin, to which none were resistant. However, for one isolate, the MIC for erythromycin $(2 \mathrm{mg} / \mathrm{L})$, and for another, the MIC for penicillin $(0.25 \mathrm{mg} / \mathrm{L})$, was above the breakpoint for S. agalactiae. Additionally, bimodal distributions of MIC were observed for fusidic acid and oxacillin. For $S$. uberis, there are no cut-off values, but distributions of MICs for some antibiotics were trimodal (erythromycin, penicillin, and tetracycline) or bimodal (cefotaxime, clindamycin, gentamicin, kanamycin, and oxacillin), indicating various degrees of acquired resistance in some isolates (Table 3). Seventeen isolates had deviating high MICs for penicillin above $0.06 \mathrm{mg} / \mathrm{L}$, of which one isolate had $\mathrm{MIC}=4 \mathrm{mg} / \mathrm{L}$ (Table 3).

Among E. coli, 17 isolates (14.7\%) were resistant to one or more antibiotics. Ten isolates $(8.6 \%)$ were resistant to at least two, and $8(6.9 \%)$ to three or more antibiotics (Table 4$)$. The most common traits were resistance to ampicillin $(8.6 \%)$, streptomycin $(7.8 \%)$, or sulphonamides (6.9\%) (Supplementary Table S2). Six isolates (5.2\%) were resistant to all these antibiotics. Five (4.3\%) isolates were resistant to ceftazidime, but none of the isolates displayed phenotypic evidence of the production of ESBLs and hence were not put forward for genotypic testing. One isolate was resistant to ciprofloxacin while susceptible to nalidixic acid and was hence tested for the presence of plasmid-borne quinolone resistance genes but was confirmed as negative by PCR. For Klebsiella, most isolates were resistant to ampicillin (95.4\%) and a few also to tetracycline $(9.1 \%)$, colistin $(4.6 \%)$, or ciprofloxacin (4.6\%) (Table 4). One isolate had elevated MIC for colistin (4 mg/L), florfenicol (32 mg/L), chloramphenicol (32 mg/L), nalidixic acid (16 mg/L), streptomycin $(64 \mathrm{mg} / \mathrm{L})$, and trimethoprim $(4 \mathrm{mg} / \mathrm{L})$. Bimodal distributions of MIC values indicate acquired resistance in some isolates to chloramphenicol, florfenicol, nalidixic acid, streptomycin, sulphamethoxazole, and trimethoprim. None of the isolates were resistant to 3rd generation cephalosporins, indicating that none produced ESBLs.

EUCAST ECOFFs have not been defined for T. pyogenes, but the MIC distributions indicate that most isolates had not acquired resistance to most of the tested antibiotics (Table 5). Exceptions from this were trimethoprim alone and in combination with sulphamethoxazole as well as tetracycline, where bimodal distributions were seen. Two isolates had deviating high MICs for penicillin over $0.06 \mathrm{mg} / \mathrm{L}$.

\subsection{Risk Factors}

Potential risk factor variables (Supplementary Table S1) contained between 0 and 25\% missing values, after excluding the variable udder disease score due to a large proportion of missing records $(67 \%)$. All missing values were successfully imputed, and comparison of diagnostic plots and tabulation of imputed and original values showed good imputation. We used pseudo-R-squared to compare the predictive abilities of the CC and MI models (Supplementary Tables S3 and S4). In general, the two models produced similar results and had the same subset of risk factors, but when comparing their predictive abilities, CC was superior to the MI model for all outcomes except $S$. aureus, for which it made no difference. Both results are presented in the tables but only the results from the CC analysis will be 
discussed. For both S. uberis and NAS, none of the significant risk factors from the MI model had missing values, and hence CC analysis was performed on all records.

Risk factor analysis was performed on data on the microbial panorama from cows with clinical mastitis, and the results must therefore be interpreted accordingly. Hence, a significant risk factor is associated with a higher likelihood of isolating a certain pathogen, given that a cow is infected. Results from the final multivariable models, including both CC and MI, can be found in Supplementary Table S3 for S. aureus, T. pyogenes and E. coli and in Supplementary Table S4 for S. dysgalactiae, S. uberis, Klebsiella spp., and NAS. The variables year, parity, breed, antibiotic treatment during the previous 30 days, dry cow antibiotic treatment at the latest dry off, and AMS were not significant in any of the final models.

Samples were accepted from all regions of Sweden (Supplementary Table S1). However, it became clear that some regions were over-represented and others under-represented considering the distribution of dairy cows in the country [19]. Calculations of regional effects were therefore not taken into consideration.

The risk of isolating $S$. aureus was higher if the cow was housed in tie stalls rather than loose housing and if the cow was in early compared with mid lactation. S. aureus was also more common in quarters with two or more udder pathogens isolated compared with only one and in mastitis cases occurring during the late housing season (January to April) compared with the early housing season (September to December). The probability of isolating NAS was higher during the late housing and pasture (May to August) seasons than during early housing season.

Streptococcus dysgalactiae was more common in quarters with two or more udder pathogens and from cases in early and peak rather than in mid lactation. The probability of isolating S. uberis was higher in cases during the early rather than the late housing season.

The risk of isolating E. coli was higher in peak and mid lactation compared with early lactation but was less common in quarters with two or more udder pathogens. Klebsiella spp. was 3.6 times more common in cows that had had a previous case of clinical mastitis in the current lactation, but it was also more rarely isolated in the early housing season than during the pasture season.

Trueperella pyogenes was more frequently found together with one or two other udder pathogens than in isolation and was also more common in cows in early lactation than in later lactation stages. It was also more common if the cow was in loose housing than in tie stalls and if the cow had a previous case of clinical mastitis in the current lactation.

\section{Discussion}

By comparing reports from different countries, it seems as though each country or study has its own unique panorama. For example, clinical cases in Finnish and Canadian dairy farms are often caused by NAS species [20,21], whereas in Sweden, very few clinical cases were due to this group of bacteria. Additionally, in many other countries, environmental pathogens such as E. coli or S. uberis dominate, while S. aureus is less common [22-25]. Whether the differences between countries are related to management practices, such as the use/non-use of blanket dry cow therapy, or other factors is currently not known. However, the five most common pathogens in clinical mastitis, S. aureus, E. coli, S. dysgalactiae, S. uberis and NAS, are seen in most studies, including ours [12].

Compared with the latest Swedish surveys [7,9], small but significant differences in the panorama were demonstrated with a relatively larger proportion of $S$. aureus, smaller proportions of NAS and samples with no growth in the present study. The dominance of the species S. epidermidis, S. chromogenes, S. simulans and S. haemolyticus among the NAS species confirms results from other studies [26-29]. Interestingly, the proportion of samples with no growth was also substantially lower than in many other reports $[20,21,30,31]$. There are several reasons for no growth in clinical mastitis samples; it has, for example, been suggested that some cases are due to E. coli infections that have been cleared by the cow's immune system [32] and that the freezing of milk samples may have a negative effect 
on the survival and growth of certain bacteria such as E. coli but not S. aureus [33]. Since this pathogen is less commonly isolated in Sweden than elsewhere, fewer samples may also be culture negative. The likelihood of a negative culture also depends on the stage of the infection and inflammation. In our investigation, only clinical cases were included, which may have increased the likelihood of a positive culture. Another hypothesis is that some of the no growth samples in other studies may be due to Mycoplasma bovis infections, a pathogen that is not detected with routine culture methods. However, recent studies indicate that M. bovis is uncommon in Swedish dairy herds [34].

Udder pathogens were isolated from milk samples collected from all parts of Sweden. However, over half of the cases were from the northern parts of Sweden, although the southern parts are the most densely cow-populated areas [19]. This is a weakness of the present study. However, the distribution of mastitis-causing pathogens was similar to the previous nationwide survey in Sweden, in which a representative sample of cows from all parts of Sweden was included during the period 2002-2003 [9]. We therefore believe that our results are representative of the real panorama of pathogens causing clinical mastitis in Swedish dairy herds, although the samples were not geographically evenly distributed over the country. The veterinarians were instructed to submit a sample from the first cases of mastitis they were called to in the month, and this was done to avoid any selection bias. On the other hand, when more than one quarter displayed clinical mastitis, they were instructed to sample the quarter showing the most severe symptoms. This was to increase the chance of detecting a pathogen during laboratory examination, but since, in most cases, clinical mastitis in more than one quarter of the same cow at the same time is caused by the same pathogen, this will have had little influence on the results.

Benzylpenicillin is the drug of choice for most cases of mastitis in Sweden, and most Gram-positive udder pathogens are also susceptible to this drug. In the present study, 2.6\% of $S$. aureus were resistant to penicillin. This is lower (results not shown) than in the latest nationwide surveys in 2002-2003 where penicillin resistance was seen in 7.1\% of isolates [8]. Comparisons between studies should be made with caution, but the results indicate that penicillin-resistant $S$. aureus are on the decline in Sweden [7,9]. This may be an effect of the recommendation to cull all cows with penicillin-resistant $S$. aureus. A decline in penicillin resistance in S. aureus was also recently reported from France during the period 2006-2016, although the proportion of resistant isolates (33.9\%) was considerably higher than in our study [35]. Considerably higher levels of resistance to penicillin in S. aureus have also been reported from several other countries [36,37].

Although occurrence of penicillin resistance in NAS in the 2002-2003 study (12.5\%) [8] was numerically lower than in the present study (30.4\%), the difference was not statistically different (results not shown), and the limited number of isolates tested precludes conclusions on trends. On the other hand, the proportion of resistant NAS resembles that detected by Persson et al. [38] from subclinical mastitis cases in Sweden. Methicillin resistance was not found in any of the staphylococci, indicating that methicillin-resistant $S$. aureus (MRSA) are rare in cases of clinical mastitis, and hitherto, only ten sporadic cases have been confirmed as MRSA in Swedish dairy cows [39]. Keeping the cattle population free from MRSA and LA-MRSA depends on hygiene measures and biosecurity. In a recent study in Denmark, it was demonstrated that LA-MRSA in cattle herds-both dairy and veal—were caused by spill-over from pig production [40]. However, the Swedish pig herds are free of LA-MRSA, which eliminates this hazard for the cattle population. The proportion of isolates with elevated MIC values for penicillin was higher for the NAS isolates than for $S$. aureus. This is in agreement with the findings from a recent German study [41] and in a study of isolates from nine different EU countries [42]. With only 21 NAS isolates, our data are insufficient to determine any differences in resistance or MIC distributions between different NAS species. It can only be encouraged that such issues are taken up by VETCAST or other fora, so that proper MIC distributions and ECOFFs for the most important NAS can be established, e.g., for S. chromogenes, S. simulans, or S. haemolyticus. Only very few ECOFFs or TECOFFs are available for these species. 
All S. agalactiae and the majority of $S$. dysgalactiae and S. uberis had low MICs to penicillin, indicating clinical susceptibility, but one isolate of $S$. dysgalactiae and seventeen of S. uberis had MICs $0.12-0.25 \mathrm{mg} / \mathrm{L}$, which indicates some degree of acquired resistance. Although there seems to be a trend towards decreased susceptibility of S. uberis to some antibiotics, penicillin and erythromycin since the last survey [8], the proportion of deviating high MICs is still much lower than in other European countries [43]. The distribution of MICs for S. uberis was trimodal, and it has been shown that mutations in penicillin-binding protein gene 1 in S. uberis can be evoked by repeated exposure to penicillin, resulting in a manifold increase in MIC [6]. The same mutations have been demonstrated in isolates from clinical bovine mastitis [6]. The existence of isolates with elevated MIC values for penicillin has also been reported by other researchers [28,41,42]. Still, most isolates have MIC values below the concentrations obtained in the inflamed udder using the pharmaceutically recommended dosage [44]. However, one isolate had MIC of $4 \mathrm{mg} / \mathrm{L}$, which was much higher than in any of the isolates from a European study [43]. It is currently unknown whether such strains with elevated penicillin MIC values might cause therapy failure, and further research and monitoring of penicillin susceptibility of $S$. uberis is therefore critical.

The proportion of E. coli resistant to quinolones was lower (1.7\%) than in the last survey (3.1\%) [8]. Although we cannot exclude that this may in part be attributed to the uneven geographical origin of the samples, a possible explanation is a change in prescription. In Sweden, the use of critically important antibiotics, including fluoroquinolones, for animals was strictly regulated in 2013 [45]. A susceptibility test showing that no other antibiotics are effective is now needed to prescribe these drugs in non-life-threatening cases of mastitis. This is probably the reason why the prescription of fluoroquinolones has decreased and the use of trimethoprim-sulphonamide combinations has increased at around the same time [2]. The proportion of isolates resistant to more than one antibiotic was numerically higher in the present survey, but comparison is questionable due to the different sets of antibiotics tested. Neither among Klebsiella spp. nor E. coli was it possible to demonstrate ESBL production. Only a few isolates of ESBL-producing E. coli from cattle origin have been confirmed in Sweden, and none were from udder infections, a situation that differs from many other countries [46]. Klebsiella is considered intrinsically resistant to ampicillin [47], but apart from this, most isolates were susceptible to other compounds.

There are no approved epidemiological breakpoints for T. pyogenes, which makes conclusions about susceptibility more difficult to reach. Most isolates had MIC values below the concentration of benzylpenicillin obtained in the inflamed udder using the pharmaceutically recommended dosage, indicating clinical susceptibility. Bimodal distributions to some antibiotics were seen, but overall, MIC values for T. pyogenes were low, which is in accordance with surveys in other countries [48-50]. However, in vitro susceptibility is not the same as in vivo, and it may still not be possible to obtain clinical cure with this antibiotic [51].

Overall, the results of the susceptibility tests are similar to the previous survey [8], and there are no other indications of major shifts in susceptibility. This is in accordance with conclusions by Oliver and Murinda [36]. These authors compared the results from several studies of antibiotic resistance in mastitis pathogens from different countries and concluded that although antibiotic use in dairy cows can contribute to increased resistance, they found no indications of any widespread emergence of resistance in mastitis pathogens.

Housing seemed to be an important factor in determining the microbial panorama. We found that $S$. aureus was associated with tie stall housing, which is in line with previous observations $[9,31]$. These authors also found E. coli to be more common in loose housing [9,31] and S. uberis in tie stalls [31], which could not be confirmed by the present investigation. On the other hand, we found that $T$. pyogenes was more common in loose housing. Given these results combined, it is likely that the distribution of udder pathogens will change if we move towards more loose housing herds in Sweden, but hitherto, major shifts have not been observed. 
Another factor that plays an important role is the season of sampling, which affected $S$. aureus, S. uberis, Klebsiella spp., and NAS. The finding that NAS and S. aureus mastitis was more common during the late housing season confirmed the results from Koivula et al. [20]. Studies in Norway [52] and Canada [53] also found that NAS was more likely during the late indoor season. In a study by Østerås et al. [52], S. aureus was most prevalent during the summer months, which is in contrast with the observations from our previous Swedish study [9], which related the higher isolation rate during the winter season to increased transmission indoors. However, with more loose housing, in which the transmission patterns are more similar throughout the year, it is more difficult to attribute seasonal differences to housing. Additionally, we could not see a sharp line between the indoor and pasture season regarding $S$. aureus isolation, and seasonal effects may instead be due to differences in climatic conditions over the year, which was also suggested by Koivula et al. [20]. Season also affected the probability of isolating S. uberis, and we saw a lower likelihood during the early housing season compared with the pasture and late housing season. This is in line with Lundberg et al. [54] and Makovec and Ruegg [55] but differs from other reports on seasonal differences for S. uberis $[8,18,52,53]$. Klebsiella spp. was more common during the pasture (summer) season, similar to previous findings [55]. Compared with the winter indoor season, during the summer, cows are more affected by adverse weather and sudden weather changes such as thunderstorms, which have been shown to predispose to coliform mastitis outbreaks [56]. Additionally, Klebsiella spp. seem to thrive better in bedding material during the summer compared with the winter [57]. We have no firm explanation as to why seasonal effects differ between different studies, and there may be other confounding factors that are masked by season, such as climate or whether cows are housed indoors or outdoors.

Consistent with the results of Persson Waller et al. [58], S. aureus and T. pyogenes peaked in early lactation, and we assume that these intramammary infections are predominantly persistent rather than new infections. It has been shown that many cows with $S$. aureus intramammary infection in early lactation were already infected at drying off, some despite treatment with dry cow antibiotics [59]. Cows are often immunocompromised around parturition [60], and it may be speculated that this may precipitate a persistent, subclinical infection into clinical mastitis. Trueperella pyogenes is often referred to as "dry cow mastitis", and infection occurred more readily and with more severe clinical signs during the dry period than during lactation in an experimental study [61]. In the same study, the infection was rarely eliminated during the dry period and often persisted into the next lactation. In contrast, we found that $E$. coli was most commonly isolated in peak lactation, which is in line with Persson Waller et al. [58]. Likewise, the risk of E. coli infection seems to increase with milk yield [62]. The probability of $S$. dysgalactiae infection has been reported to decrease with increasing lactation stage [52], but in the present study, it occurred more commonly in early and peak than in mid lactation, which confirms previous results [58].

Both Klebsiella spp. and T. pyogenes were over three times more likely to be isolated in cows that had had a previous case of clinical mastitis. It can be assumed that a case of clinical mastitis makes the quarter more susceptible to a new intramammary infection [63]. Additionally, T. pyogenes may in some cases require a concomitant infection with another pathogen to cause clinical signs $[61,64]$. Likewise, we found that T. pyogenes was almost 18 times more likely to be isolated together with another pathogen. Additionally, S. dysgalactiae and S. aureus were more likely to be isolated together with another bacterium than others, and the results are in line with the previous survey [9]. These three bacteria were the only combination seen in quarters with three udder pathogens in the present study (results not shown). Co-infections of $S$. dysgalactiae and T. pyogenes are commonly seen in quarters from Swedish cows with mastitis [54,64], as are combinations of S. aureus and S. dysgalactiae [54]. Escherichia coli, on the other hand, was more often found in monoculture in this study, which is in contrast with the previous Swedish survey [9]. 


\section{Conclusions}

The most common pathogens found in cases of clinical mastitis were S. aureus, E. coli, S. dysgalactiae and S. uberis. In general, these pathogens were mostly susceptible to antibiotics commonly used in Sweden. Very few S. aureus were resistant to penicillin, while some isolates of $S$. uberis had an elevated MIC value due to penicillin, something that may offer therapeutic challenges. Housing, season, and history of previous cases of clinical mastitis were all identified as risk factors, depending on bacterial species.

Supplementary Materials: The following are available online at https:/ /www.mdpi.com/article/10 $.3390 /$ ani11072113/s1, Table S1: Descriptive statistics of variables reflecting farm and cow characteristics, Table S2: Resistance phenotypes of Escherichia coli $(n=17)$ resistant to at least one antibiotic, Table S3: Final multivariate models with odds ratio (OR) estimates with $95 \%$ confidence intervals (CI) for the risk of isolating Staphylococcus aureus, Trueperella pyogenes, and Escherichia coli from cases of clinical mastitis in 653 Swedish dairy cows, Table S4: Final multivariate models with odds ratio (OR) estimates with $95 \%$ confidence intervals (CI) for the risk of isolating Streptococcus dysgalactiae, Streptococcus uberis, Klebsiella spp. and non-aureus staphylococci (NAS) from cases of clinical mastitis in 653 Swedish dairy cows.

Author Contributions: Conceptualization, K.P. and K.P.-W.; methodology, A.D. and K.P.-W; software, A.D.; validation, K.P., K.P.-W. and A.D.; formal analysis, A.D.; resources, K.P.; data curation, A.D.; writing—original draft preparation, A.D.; writing—review and editing, K.P., K.P.-W. and A.D.; visualization, A.D.; supervision, K.P.; project administration, K.P.; funding acquisition, K.P. All authors have read and agreed to the published version of the manuscript.

Funding: This research was funded by the Swedish Board of Agriculture through the SvarmPat programme.

Institutional Review Board Statement: Ethical review and approval was waived for this study since sampling of clinical material from diseased animals for laboratory examination and diagnosis is not considered animal experiment but a part of ordinary veterinary treatment practices. These samples do not require ethical approvement, according to Swedish (SJVFS 2019:9, chapter 2 § 17-18) and EU legislations (2010/63/EU, article 1:5: "practices not likely to cause pain, suffering, distress or lasting harm equivalent to, or higher than, that caused by the introduction of a needle in accordance with good veterinary practice").

Informed Consent Statement: Not applicable.

Data Availability Statement: The datasets generated and analysed during this study are available upon reasonable request. Bacterial isolates are available and can be requested provided an MTA is agreed.

Acknowledgments: The Swedish District Veterinary Organisation is gratefully acknowledged for collecting and submitting mastitis samples. Technical staff at SVA is gratefully acknowledged for doing the laboratory analyses. We are also grateful for the contribution by Helle Ericsson Unnerstad (17 June 1968-11 March 2019) who sadly passed away during this investigation.

Conflicts of Interest: The authors declare no conflict of interest.

\section{References}

1. Jamali, H.; Barkema, H.W.; Jacques, M.; Lavallée-Bourget, E.M.; Malouin, F.; Saini, V.; Stryhn, H.; Dufour, S. Invited review: Incidence, risk factors, and effects of clinical mastitis recurrence in dairy cows. J. Dairy Sci. 2017, 101, 4729-4746. [CrossRef]

2. Redogörelse för Husdjursorganisationernas Djurhälsovård 2017-2018; Växa Sverige: Stockholm, Sweden, 2019.

3. Acar, J.; Röstel, B. Antimicrobial resistance: An overview. Rev. Sci. Tech. Int. Off. Epizoot. 2001, 20, 797-810. [CrossRef]

4. Mader, R.; Damborg, P.; Amat, J.-P.; Bengtsson, B.; Bourély, C.; Broens, E.M.; Busani, L.; Crespo, P.; Filippitzi, M.-E.; Fitzgerald, W.; et al. Building the European Antimicrobial Resistance Surveillance network in veterinary medicine (EARS-Vet). Eurosurveillance 2021, 26. [CrossRef]

5. Persson Waller, K.; Hårdemark, V.; Nyman, A.K.; Duse, A. Veterinary treatment strategies for clinical mastitis in dairy cows in Sweden. Vet. Rec. 2016, 178, 240. [CrossRef]

6. Haenni, M.; Galofaro, L.; Ythier, M.; Giddey, M.; Majcherczyk, P.; Moreillon, P.; Madec, J.-Y. Penicillin-binding protein gene alterations in Streptococcus uberis isolates presenting decreased susceptibility to penicillin. Antimicrob. Agents Chemother. 2010, 54, 1140-1145. [CrossRef] [PubMed] 
7. Nilsson, L.; Franklin, A.; Funke, H. Antimicrobial drug susceptibility of bovine udder pathogens in Sweden. In Proceedings of the 15th Annual Meeting Society Veterinary Epidemiology Preventive Medicine, Chester, UK, 9-11 April 1997.

8. Bengtsson, B.; Unnerstad, H.E.; Ekman, T.; Artursson, K.; Nilsson-Öst, M.; Waller, K.P. Antimicrobial susceptibility of udder pathogens from cases of acute clinical mastitis in dairy cows. Vet. Microbiol. 2009, 136, 142-149. [CrossRef] [PubMed]

9. Ericsson Unnerstad, H.; Lindberg, A.; Persson Waller, K.; Ekman, T.; Artursson, K.; Nilsson-Öst, M.; Bengtsson, B. Microbial aetiology of acute clinical mastitis and agent-specific risk factors. Vet. Microbiol. 2009, 137, 90-97. [CrossRef]

10. Brolund, L. Technical Utilization of Cell Count in the Milk-Recording Service (Cellhaltens Tekniska Utnyttjande i Kokontrollen); Swedish Association for Livestock Breeding and Production: Eskilstuna, Sweden, 1990; pp. 40-42.

11. European Union. Regions in the European Union-Nomenclature of Territorial Units for Statistics-NUTS 2010/EU-27; Eurostat: Luxemburg, 2011.

12. Nonnemann, B.; Svennesen, L.; Lyhs, U.; Kristensen, K.A.; Klaas, I.C.; Pedersen, K. Bovine mastitis bacteria resolved by matrix-assisted laser desorption/ionization time of flight mass spectrometry. J. Dairy Sci. 2019, 102, 2515-2524. [CrossRef]

13. Performance Standards for Antimicrobial Disk and Dilution Susceptibility Tests for Bacteria Isolated from Animal: Approved Standards. CLSI Document VET01-A4, 4th ed.; Clinical and Laboratory Standards Institute (CLSI): Wayne, PA, USA, 2013.

14. Bryan, L.E.; Godfrey, A.J. Beta-Lactam Antibiotics: Mode of Action and Bacterial Resistance. In Antibiotics in Laboratory Medicine; Lorian, V., Ed.; William \& Wilkins: Baltimore, MD, USA, 1991; pp. 599-604.

15. Pichon, B.; Hill, R.; Laurent, F.; Larsen, A.R.; Skov, R.L.; Holmes, M.; Edwards, G.F.; Teale, C.; Kearns, A.M. Development of a real-time quadruplex PCR assay for simultaneous detection of nuc, Panton-Valentine leucocidin (PVL), mecA and homologue mecALGA251. J. Antimicrob. Chemother. 2012, 67, 2338-2341. [CrossRef] [PubMed]

16. Cavaco, L.M.; Frimodt-Møller, N.; Hasman, H.; Guardabassi, L.; Nielsen, L.; Aarestrup, F.M. Prevalence of quinolone resistance mechanisms and associations to minimum inhibitory concentrations in quinolone-resistant Escherichia coli isolated from humans and swine in Denmark. Microb. Drug Resist. 2008, 14, 163-169. [CrossRef]

17. Dohoo, I.R.; Nielsen, C.R.; Emanuelson, U. Multiple imputation in veterinary epidemiological studies: A case study and simulation. Prev. Vet. Med. 2016, 129, 35-47. [CrossRef] [PubMed]

18. Hosmer, D.W.; Lemeshow, S. Applied Logistic Regression; Wiley: New York, NY, USA, 2013.

19. Agricultural Statistics 2017 Including Food Statistics-Tables; Statistics Sweden: Örebro, Sweden, 2017.

20. Koivula, M.; Pitkälä, A.; Pyörälä, S.; Mäntysaari, E.A. Distribution of bacteria and seasonal and regional effects in a new database for mastitis pathogens in Finland. Acta Agric. Scand. Sect. Anim. Sci. 2007, 57, 89-96. [CrossRef]

21. Levison, L.J.; Miller-Cushon, E.K.; Tucker, A.L.; Bergeron, R.; Leslie, K.E.; Barkema, H.W.; DeVries, T.J. Incidence rate of pathogen-specific clinical mastitis on conventional and organic Canadian dairy farms. J. Dairy Sci. 2016, 99, 1341-1350. [CrossRef] [PubMed]

22. Milne, M.H.; Barret, D.C.; Fitzpatrick, J.L.; Biggs, A.M. Prevalence and aetiology of clinical mastitis on dairy farms in Devon. Vet. Rec. 2002, 151, 241-243. [CrossRef]

23. Botrel, M.-A.; Haenni, M.; Morignat, E.; Sulpice, P.; Madec, J.-Y.; Calavas, D. Distribution and antimicrobial resistance of clinical and subclinical mastitis pathogens in dairy cows in Rhône-Alpes, France. Foodborne Pathog. Dis. 2009, 7, 479-487. [CrossRef]

24. Oliveira, L.; Hulland, C.; Ruegg, P.L. Characterization of clinical mastitis occurring in cows on 50 large dairy herds in Wisconsin. J. Dairy Sci. 2013, 96, 7538-7549. [CrossRef]

25. Gao, J.; Barkema, H.W.; Zhang, L.; Liu, G.; Deng, Z.; Cai, L.; Shan, R.; Zhang, S.; Zou, J.; Kastelic, J.P.; et al. Incidence of clinical mastitis and distribution of pathogens on large Chinese dairy farms. J. Dairy Sci. 2017, 100, 4797-4806. [CrossRef] [PubMed]

26. Persson Waller, K.; Aspan, A.; Nyman, A.; Persson, Y.; Grönlund Andersson, U. CNS species and antimicrobial resistance in clinical and subclinical bovine mastitis. Vet. Microbiol. 2011, 152, 112-116. [CrossRef] [PubMed]

27. Mahmmod, Y.; Nonnemann, B.; Svennesen, L.; Pedersen, K.; Klaas, I. Typeability of MALDI-TOF assay for identification of non-aureus staphylococci associated with bovine intramammary infections and teat apex colonization. J. Dairy Sci. 2018, 101, 9430-9438. [CrossRef]

28. Chehabi, C.N.; Nonnemann, B.; Astrup, L.B.; Farre, M.; Pedersen, K. In vitro antimicrobial resistance of causative agents to clinical mastitis in dairy cows. Foodborne Pathog. Dis. 2019, 15, 562-572. [CrossRef] [PubMed]

29. Hamel, J.; Zhang, Y.; Wente, N.; Krömker, V. Non-S. aureus staphylococci (NAS) in milk samples: Infection or contamination? Vet. Microbiol. 2020, 242, 108594. [CrossRef]

30. Bradley, A.J.; Leach, K.A.; Breen, J.E.; Green, L.E.; Green, M.J. Survey of the incidence and aetiology of mastitis on dairy farms in England and Wales. Vet. Rec. 2007, 160, 253-258. [CrossRef]

31. Riekerink, R.G.M.O.; Barkema, H.W.; Kelton, D.F.; Scholl, D.T. Incidence rate of clinical mastitis on Canadian dairy farms. J. Dairy Sci. 2008, 91, 1366-1377. [CrossRef]

32. Shinozuka, Y.; Morita, T.; Watanabe, A.; Kawai, K. Live bacteria in clots from bovine clinical mastitis milk with no growth in conventional culturing. Asian J. Anim. Vet. Adv. 2018, 13, 197-200. [CrossRef]

33. Hubackova, M.; Rysánek, D. Effects of freezing milk samples on the recovery of alimentary pathogens and indicator microorganisms. Acta Vet. Brno 2007, 76, 301-307. [CrossRef]

34. Swedish University of Agricultural Sciences. Low Prevalence of Mycoplasma bovis in Sweden. Available online: https: / / www.slu.se/ew-nyheter/2020/3/mjolktankar (accessed on 14 July 2021). 
35. Boireau, C.; Cazeau, G.; Jarrige, N.; Calavas, D.; Madec, J.-Y.; Leblond, A.; Haenni, M.; Gay, E. Antimicrobial resistance in bacteria isolated from mastitis in dairy cattle in France, 2006-2016. J. Dairy Sci. 2018, 101, 9451-9462. [CrossRef]

36. Oliver, S.P.; Murinda, S.E. Antimicrobial resistance of mastitis pathogens. Vet. Clin. Food Anim. 2012, 28, 165-185. [CrossRef]

37. Bolte, J.; Zhang, Y.; Wente, N.; Mahmmod, Y.S.; Svennesen, L.; Krömker, V. Comparison of phenotypic and genotypic antimicrobial resistance patterns associated with Staphylococcus aureus in German and Danish dairy cows. J. Dairy Sci. 2020, 103, 3554-3564 [CrossRef] [PubMed]

38. Persson, Y.; Nyman, A.-K.J.; Grönlund-Andersson, U. Etiology and antimicrobial susceptibility of udder pathogens from cases of subclinical mastitis in dairy cows in Sweden. Acta Vet. Scand. 2011, 53, 36. [CrossRef] [PubMed]

39. Unnerstad, H.E.; Mieziewska, K.; Börjesson, S.; Hedbäck, H.; Strand, K.; Hallgren, T.; Landin, H.; Skarin, J.; Bengtsson, B. Suspected transmission and subsequent spread of MRSA from farmer to dairy cows. Vet. Microbiol. 2018, 225, 114-119. [CrossRef]

40. Hansen, J.E.; Ronco, T.; Stegger, M.; Sieber, R.N.; Fertner, M.E.; Martin, H.L.; Farre, M.; Toft, N.; Larsen, A.R.; Pedersen, K. MRSA CC398 in dairy cattle and veal calf farms indicates spillover from pig production. Front. Microbiol. 2019, 10, 2733. [CrossRef] [PubMed]

41. Bolte, J.; Zhang, Y.; Wente, N.; Krömker, V. In vitro susceptibility of mastitis pathogens isolated from clinical mastitis cases in Northern German dairy farms. Vet. Sci. 2020, 7, 10. [CrossRef] [PubMed]

42. De Jong, A.; Garch, F.E.; Simjee, S.; Moyaert, H.; Rose, M.; Youala, M.; Siegwart, E.; VetPath Study Group. Monitoring of antimicrobial susceptibility of udder pathogens recovered from cases of clinical mastitis in dairy cows across Europe: VetPath results. Vet. Microbiol. 2018, 213, 73-81. [CrossRef]

43. Thomas, V.; de Jong, A.; Moyaert, H.; Simjee, S.; El Garch, F.; Morrissey, I.; Marion, H.; Vallé, M. Antimicrobial susceptibility monitoring of mastitis pathogens isolated from acute cases of clinical mastitis in dairy cows across Europe: VetPath results. Int. J. Antimicrob Agents 2015, 46, 13-20. [CrossRef]

44. Franklin, A.; Olof, H.; Rantzien, M.H.; Aström, G. Effect of procaine benzylpenicillin alone or in combination with dihydrostreptomycin on udder pathogens in vitro and in experimentally infected bovine udders. Am. J. Vet. Res. 1984, 45, 1398-1402.

45. Statens Jordbruksverks Föreskrifter om Läkemedel och Läkemedelsanvändning; Swedish Board of Agriculture: Jönköping, Sweden, 2013.

46. Dantas Palmeira, J.; Ferreira, H.M.N. Extended-spectrum beta-lactamase (ESBL)-producing Enterobacteriaceae in cattle production-A threat around the world. Heliyon 2020, 6. [CrossRef]

47. Bernardini, A.; Cuesta, T.; Tomas, A.; Bengoechea, J.A.; Martínez, J.L.; Sanchez, M.B. The intrinsic resistome of Klebsiella pneumoniae. Int. J. Antimicrob. Agents. 2019, 53, 29-33. [CrossRef] [PubMed]

48. Jousimies-Somer, H.; Pyörälä, S.; Kanervo, A. Susceptibilities of bovine summer mastitis bacteria to antimicrobial agents. Antimicrob. Agents Chemother. 1996, 40, 157-160. [CrossRef]

49. Alkasir, R.; Wang, J.; Gao, J.; Ali, T.; Zhang, L.; Szenci, O.; Bajcsy, Á.C.; Han, B. Properties and antimicrobial susceptibility of Trueperella pyogenes isolated from bovine mastitis in China. Acta Vet. Hung. 2016, 64, 1-12. [CrossRef]

50. Galán-Relaño, Á.; Gómez-Gascón, L.; Barrero-Domínguez, B.; Luque, I.; Jurado-Martos, F.; Vela, A.I.; Sanz-Tejero, C.; Tarradas, C. Antimicrobial susceptibility of Trueperella pyogenes isolated from food-producing ruminants. Vet. Microbiol. 2020, $242,108593$. [CrossRef]

51. Waage, S.; Skei, H.R.; Rise, J.; Rogdo, T.; Sviland, S.; Ødegaard, S.A. Outcome of clinical mastitis in dairy heifers assessed by reexamination of cases one month after treatment. J. Dairy Sci. 2000, 83, 70-76. [CrossRef]

52. Østerås, O.; Sølverød, L.; Reksen, O. Milk culture results in a large Norwegian survey—effects of season, parity, days in milk, resistance, and clustering. J. Dairy Sci. 2006, 89, 1010-1023. [CrossRef]

53. Riekerink, R.G.M.O.; Barkema, H.W.; Stryhn, H. The effect of season on somatic cell count and the incidence of clinical mastitis. J. Dairy Sci. 2007, 90, 1704-1715. [CrossRef] [PubMed]

54. Lundberg, Å.; Nyman, A.-K.; Aspán, A.; Börjesson, S.; Unnerstad, H.E.; Waller, K.P. Udder infections with Staphylococcus aureus, Streptococcus dysgalactiae, and Streptococcus uberis at calving in dairy herds with suboptimal udder health. J. Dairy Sci. 2016, 99, 2102-2117. [CrossRef]

55. Makovec, J.A.; Ruegg, P.L. Results of milk samples submitted for microbiological examination in Wisconsin from 1994 to 2001. Dairy Sci. 2003, 86, 3466-3472. [CrossRef]

56. Simensen, E. The relationship between weather and incidence of parturient paresis and mastitis in dairy cows. Nord. Vet. Med. 1974, 26, 382-386.

57. Hogan, J.S.; Smith, K.L.; Hoblet, K.H.; Todhunter, D.A.; Schoenberger, P.S.; Hueston, W.D.; Pritchard, D.E.; Bowman, G.L.; Heider, L.E.; Brockett, B.L.; et al. Bacterial counts in bedding materials used on nine commercial dairies. J. Dairy Sci. 1989, 72, 250-258. [CrossRef]

58. Persson Waller, K.; Bengtsson, B.; Lindberg, A.; Nyman, A.; Ericsson Unnerstad, H. Incidence of mastitis and bacterial findings at clinical mastitis in Swedish primiparous cows- influence of breed and stage of lactation. Vet. Microbiol. 2009, 134, 89-94. [CrossRef]

59. Buddle, B.M.; Cooper, M.G. Dry-cow therapy for Staphylococcus aureus mastitis. N. Z. Vet. J. 1980, 28, 51-53. [CrossRef]

60. Wankhade, P.R.; Manimaran, A.; Kumaresan, A.; Jeyakumar, S.; Ramesha, K.P.; Sejian, V.; Rajendran, D.; Varghese, M.R. Metabolic and immunological changes in transition dairy cows: A review. Vet. World 2017, 10, 1367-1377. [CrossRef] [PubMed]

61. Hillerton, J.E.; Bramley, A.J. Infection following challenge of the lactating and dry udder of dairy cows with Actinomyces pyogenes and Peptostreptococcus indolicus. Br. Vet. J. 1989, 145, 148-158. [CrossRef] 
62. Zhang, Z.; Li, X.P.; Yang, F.; Luo, J.Y.; Wang, X.R.; Liu, L.H.; Li, H.S. Influences of season, parity, lactation, udder area, milk yield, and clinical symptoms on intramammary infection in dairy cows. J. Dairy Sci. 2016, 99, 6484-6493. [CrossRef] [PubMed]

63. Derakhshani, H.; Fehr, K.B.; Sepehri, S.; Francoz, D.; De Buck, J.; Barkema, H.W.; Plaizier, J.C.; Khafipour, E. Invited review: Microbiota of the bovine udder: Contributing factors and potential implications for udder health and mastitis susceptibility. J. Dairy Sci. 2018, 101, 10605-10625. [CrossRef] [PubMed]

64. Schwan, O.; Holmberg, O. Heifer mastitis and dry-cow mastitis: A bacteriological survey in Sweden. Vet. Microbiol. 1979, 3, 213-226. [CrossRef] 